\title{
Strafjustiz vs. Medien und Öffentlichkeit - zwei Akteure mit gegensätzlichen Interessen
}

\author{
Eliane Welte*
}

Die Bedeutung des Öffentlichkeitsprinzips als einer der zentralen Grundsätze der schweizerischen Strafprozessordnung ist weitgehend unbestritten. Indes stellen sich im Hinblick auf dessen praktische Handhabung diverse Fragen, etwa jene nach den Voraussetzungen der Einsichtnahme Dritter in Entscheide nichtöffentlicher Verfahren. Nebst dieser Thematik beleuchtet der vorliegende Beitrag die Rolle der Medien im Hinblick auf die Tätigkeit der Strafjustiz und die Kehrseite der Medaille in Form potenzieller unerwünschter Einflüsse und Zwänge der Medienberichterstattung gegenüber dem Strafverfahren. Die Autorin thematisiert sodann die aktuelle Informationslandschaft und kommt zum Schluss, dass die Justiz nicht umhinkommt, ihren Umgang mit dem öffentlichen Informationsinteresse zu überdenken und adäquate Wege zu finden, um ihre Tätigkeit für die Rechtsunterworfenen verständlich zu machen und die nötige Akzeptanz für ihre Entscheide zu schaffen.

I. Strafrecht interessiert - nicht nur die Juristen ........................................ 202

II. Recht der Öffentlichkeit auf Information über Strafentscheide...................... 202

III. Das Recht auf Einsichtnahme in die Entscheide in der Praxis........................203

1. Strafbefehle........................................................................................ 204

2. Einstellungs- und Nichtanhandnahmeverfügungen ..................................205

IV. Die Medien als Vermittler von Informationen............................................207

V. Strafjustiz und Medien - feindliche Brüder? ............................................. 208

1. Medienpräsenz der Verfahrensbeteiligten.............................................210

2. Mediale Einflüsse auf das Strafverfahren .....................................................211

3. Informationsbeschaffung der Bevölkerung über Strafverfahren .................. 214

4. Ausblick .................................................................................... 216

VI. Öffentlichkeitsarbeit der Strafjustiz ......................................................... 217

1. Der Fall «Carlos» oder: Wie man es nicht machen sollte ............................ 219

2. Ein Öffentlichkeitskonzept tut not......................................................... 220

Zitiervorschlag: Eliane Welte, Strafjustiz vs. Medien und Öffentlichkeit - zwei Akteure mit gegensätzlichen Interessen, in: sui-generis 2017, S. 201

URL: $\quad$ sui-generis.ch/44

DOI: $\quad$ https://doi.org/10.21257/sg.44

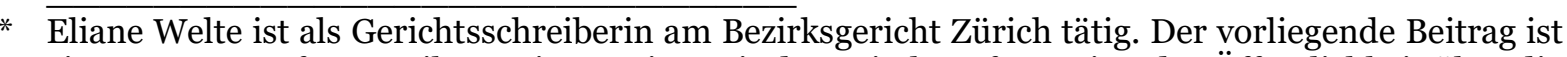
eine Zusammenfassung ihrer Dissertation mit dem Titel «Information der Öffentlichkeit über die Tätigkeit der Strafjustiz: Zur aktuellen Informationslandschaft und den Anforderungen an eine zeitgemässe Öffentlichkeitsarbeit der Strafbehörden», Diss. Univ. Zürich 2015, 2016. 


\section{Strafrecht interessiert - nicht nur die Juristen}

1 Die Tätigkeit der Strafjustiz wird von den Medien seit jeher intensiv mitverfolgt und kommentiert. Indem sie die Bevölkerung mit Informationen versorgen und - im Idealfall - Strafverfahren sachlich und kritisch begleiten, stellen die Medien ein unverzichtbares Bindeglied zwischen Justiz und Rechtsunterworfenen dar. ${ }^{1}$ Strafverfahren stossen bei der Bevölkerung auf grosses Interesse - ob aufgrund eines «echten» Informationsbedürfnisses oder einer gewissen Sensationslust ${ }^{2}$, sei dahingestellt. Dessen ungeachtet erscheint der Wunsch legitim, sich darüber ins Bild zu setzen, was bei der Strafjustiz vor sich geht. 3

\section{Recht der Öffentlichkeit auf Information über Strafentscheide}

Inbetracht der Bedeutung eines funktionierenden Justizsystems in einem demokratischen Rechtsstaat lässt sich die Notwendigkeit, die Rechtsunterworfenen über die Tätigkeit und die Entscheidungen der Justiz zu informieren, kaum bestreiten. Ein Informationsanspruch bezüglich der Entscheide der Justiz ergibt sich klar aus den gesetzlichen Grundla-

BGE 113 Ia 309 E. 4b; BVerfGE 35, 202 (222); Nobel Peter/Weber Rolf H., Medienrecht, 3. Aufl., Bern 2007, S. 144; Riklin Franz, Vorverurteilung durch die Medien, in: recht 2/1991, S. 71; Zeller Franz, Gerichtsöffentlichkeit als Quelle der Medienberichterstattung, in: medialex 1/2003, S. 16.

2 Vgl. dazu das Urteil des BGer 5A_256/2016 vom 9. Juni 2017, in welchem das Bundesgericht festhielt, dass grundsätzlich auch Sensationsberichte im öffentlichen Interesse liegen können.

3 BVerfGE 35, 202 (231); Bernhard Roberto, Gerichtsberichterstattung - Zweck und Probleme aus der Sicht der Medien, in: ZBJV 1995, S. 204; Kuss Matthias, Öffentlichkeitsmaxime der Judikative und das Verbot von Fernsehaufnahmen im Gerichtssaal, Diss. Univ. Berlin 1999, S. 69. gen, nämlich aus Art. $16 \mathrm{BV}$ in Verbindung mit Art. 30 Abs. 3 BV, welcher die Informationsfreiheit für den Bereich gerichtlicher Verfahren konkretisiert. Diese Bestimmungen sollen der Öffentlichkeit ermöglichen, die Tätigkeit der Justiz kritisch zu begleiten und dazu Stellung zu nehmen.4 Ebenso ergibt sich daraus ein Anspruch interessierter Dritter auf $\mathrm{Zu}$ gang zu den Verhandlungen und Urteilen der Gerichte. Sowohl die Gerichtsverhandlungen als auch die gefällten Urteile sind damit allgemein zugängliche Informationsquellen im Sinne der Informationsfreiheit.5

3 Das Strafprozessrecht schreibt den Grundsatz der Publikumsöffentlichkeit in Art. 69 Abs. 1 StPO fest und Abs. 2 derselben Bestimmung gewährt interessierten Dritten einen Anspruch auf Einsichtnahme in die Urteile und Strafbefehle, welche ohne öffentliche Verkündung ergangen sind. Schliesslich regelt Art. 74 StPO - im Sinne einer Durchbrechung des Grundsatzes der Geheimhaltung -

4 BGE 121 II 21 E. 4c; BVerwGE 104, 105 (109); Branahl Udo, Justizberichterstattung, eine Einführung, Wiesbaden 2005, S. 17 f.; Strebel Elisabeth, Grenzen medialer Öffentlichkeitsarbeit der Staatsanwaltschaft: zum Schutz der Persönlichkeitsrechte der beschuldigten Person im Vorverfahren, Diss. Univ. Luzern, Bern 2011, S. $25 \mathrm{f}$.

5 BGE 137 I 16 E. 2.2; BGE 139 I 129 E. 3.3; Guignard Marcel, die Gerichtsberichterstattung, in: Festschrift für den Aargauischen Juristenverein 1936-1986, Aarau 1986, S. 53 f.; Müller Jörg Paul/Schefer Markus, Grundrechte in der Schweiz, 4. Aufl., Bern 2008, S. 965; zu Recht kritisch gegenüber der Beschränkung des Öffentlichkeitsprinzips auf materielle Teil- und Endentscheide äussert sich Schindler Benjamin, Justizöffentlichkeit im digitalen Zeitalter, in: Recht im digitalen Zeitalter, Festgabe Schweizerischer Juristentag 2015 in St. Gallen, Zürich/St. Gallen 2015, S. 747. 
die Information der Öffentlichkeit über laufende Verfahren. ${ }^{6}$

\section{Das Recht auf Einsichtnahme in die Entscheide in der Praxis}

4 So klar die gesetzliche Regelung auch ist, so vielfältig sind die Hürden, auf welche Interessierte zuweilen stossen. Leider hat die Praxis des Bundesgerichts, welches seit einigen Jahren sämtliche Urteile anonymisiert im Internet publiziert, noch kaum Nachahmer gefunden.7 Im Gegenteil - wer sich für Urteile der unteren Gerichte interessiert, sieht sich häufig mit einem steinigen Hürdenlauf konfrontiert.

5 Auf gar noch mehr Widerstand stösst zuweilen, wer Einsicht in einen Entscheid in einem nichtöffentlichen Verfahren nehmen möchte, also in einen Strafbefehl oder eine Einstellungs- bzw. Nichtanhandnahmeverfügung. Bei diesen Entscheiden ist die Praxis der Justizbehörden äusserst vielfältig. ${ }^{8}$ Weder die Konzeption des Strafbefehlsverfahrens als ressourcenschonendes Prozedere für geringfügige Delikte, noch zusätzlicher

$6 \overline{\text { Oberholzer Niklaus, Grundzüge des Strafprozess- }}$ rechts, 3. Aufl., Bern 2012, Rz. 653; Saxer in BSK StPO, 2. Aufl., Basel 2014, Art. 74 Rz. 11; Strebel (Fn. 4), S. 71.

7 Flühmann Caroline/Sutter Patrick, kritische Betrachtung der bundesgerichtlichen Veröffentlichungspraxis oder «Wünschbares ist machbar», in: AJP 2003, S. 1041, sprechen sich mit Recht für eine Ausweitung der Publikation von Gerichtsurteilen im Internet aus; Schindler (Fn. 5), S. 756, kommt zum Schluss, dass insbesondere bei der kantonalen Justiz noch erhebliches Verbesserungspotenzial in Sachen Publikationspraxis besteht.

8 So die Erkundigungen von Riklin Franz, Urteilseröffnung beim Strafbefehl, in: Zen-Ruffinen Piermarco (Hrsg.), du monde pénal: droit pénal, criminoligie et politique criminelle, police et exécution des sanctions, procédure pénale: mélanges en l'honneur de Pierre-Henri Bolle, Basel 2006, S. 121. administrativer Aufwand rechtfertigen aber einen vollständigen Ausschluss Interessierter von der Einsichtnahme. Die Konsequenzen der Verweigerung der Einsichtnahme in Strafbefehle sowie Einstellungs- und Nichtanhandnahmeverfügungen sind weitreichend: Im Ergebnis bleiben damit die Entscheide in über $90 \%$ aller Strafverfahren nicht zugänglich!9 Damit verkommt die vom Gesetzgeber angestrebte Transparenz bei den Entscheiden der Strafjustiz zum reinen Wunschdenken. Durch die fehlende Einsichtsgewährung wird Interessierten die Möglichkeit genommen, auch diesen Bereich der Strafrechtspflege kritisch zu verfolgen und eine - zumindest potenzielle - Kontrollfunktion wahrzunehmen. ${ }^{10}$

6 Wird der Grundsatz der Transparenz der Rechtsprechung vom Gesetzgeber hochgehalten, Interessierten aber die Einsichtnahme in die grosse Mehrheit aller Strafentscheide verwehrt, erstaunt es wenig, dass zuweilen der Vorwurf laut wird, die Justiz wirke abgeschottet von aussen im Elfenbeinturm. Soll das Einsichtsrecht nicht leeres Versprechen bleiben, müssen auch Strafbefehle und Einstellungs- bzw. Nichtanhandnahmeverfügungen ohne hohe Hürden einsehbar sein.

9 Hutzler Doris, Ausgleich struktureller Garantedefizite im Strafbefehlsverfahren, eine Analyse der zürcherischen, schweizerischen und deutschen Regelungen, unter besonderer Berücksichtigung der Geständnisfunktion, Diss. Univ. Luzern, Baden-Baden 2010, Rz. 125; Riklin (Fn. 8), S. 115 f.; Oberholzer (Fn. 6), Rz. 1471.

10 Gless Sabine, der Strafbefehl in der Schweizerischen Strafprozessordnung, in: Heer Marianne (Hrsg.), Schweizerische Strafprozessordnung und Schweizerische Jugendstrafprozessordnung, SWR Bd. 12, Bern 2010, S. 61; Hasler Thomas, Strafverfahren - Deals verstärken den Eindruck, die Justiz sei eine Dunkelkammer, in: Tagesanzeiger Nr. 277 vom 26.11.2011, S. 13; Riklin (Fn. 8), S. 115. 


\section{Strafbefehle}

7 Wenn das Bundesgericht in seinem Leitentscheid zur Einsichtnahme in Strafbefehle $^{11}$ ein «berechtigtes Interesse» verlangt, stellt es eine sehr vage und in der Praxis kaum handhabbare Voraussetzung auf. Im Sinne der Transparenz der Rechtsprechung müsste ein blosses Interesse an Information genügen. ${ }^{12}$ Wenig konsequent erscheint, dass das Bundesgericht das Einsichtsrecht in Strafbefehle - wie bei den Strafurteilen - auf das Prinzip der öffentlichen Urteilsverkündung abstützt, für die Einsichtnahme aber höhere Hürden aufstellt. ${ }^{13}$ Im Ergebnis kommt auch das Bundesgericht zum Schluss, dass ein ernsthaftes Interesse an der Kenntnisnahme ausreiche. ${ }^{14}$ Da ein solches kaum jemandem abgesprochen werden kann, läuft diese Voraussetzung ins Leere, und es wäre nur konsequent, darauf zu verzichten. Zudem dürfte die unterschiedliche Auslegung des erforderlichen Interesses durch die Staatsanwaltschaften zu einer rechtsungleichen Behandlung Interessierter führen.

8 Bei der Einsichtnahme in Strafbefehle stellt sich indes ein zentrales praktisches Problem: Wie nämlich sollen Interessierte in einen Strafbefehl Einsicht nehmen,

11 BGE 124 IV 234.

12 So etwa auch Bommer Felix, Einstellungsverfügung und Öffentlichkeit, dogmatische Bemerkungen zum «Fall Nef», in: forumpoenale 4/2011, S. 249; Brunner Stephan, Einsichtnahme in Einstellungs- und Nichtanhandnahmeverfügungen bei Strafverfahren, Anmerkungen zu BGE 134 I 286, in: medialex 3/2008, S. 145; Saxer/Thurnheer in BSK StPO, Art. 69 Rz. 39; KGer St. Gallen, Anklagekammer, Entscheid vom 25.5.2011 (GVP 2011 Nr. 68).

13 OGer Solothurn, Beschwerdekammer, Entscheid vom 8.11.2011 (SOG 2011 Nr. 15), Bommer (Fn. 12), S. 249.

14 BGE 124 IV 234 E. 3 c. wenn sie gar keine Kenntnis von dessen Erlass haben? Aufgrund der fehlenden Hauptverhandlung müssten Interessierte regelmässig bei den Staatsanwaltschaften vorstellig werden und sich nach erlassenen Strafbefehlen erkundigen 15 - ein unzumutbarer Aufwand. Wenn das Bundesgericht ausführt, beim Erlass eines Strafbefehls genüge anstelle der Publikation dessen Auflage auf der Gerichtskanzlei bzw. die Gewährung der Einsicht auf Nachfrage eines Berechtigten hin, ${ }^{16}$ umgeht es geflissentlich die Auseinandersetzung mit dieser Problematik. Die blosse Auflage der Strafbefehle ohne Hinweis auf deren Erlass vermag dem Öffentlichkeitsprinzip nicht zu genügen. ${ }^{17}$

9 Soll auch bei den Strafbefehlen Transparenz herrschen, führt an deren Publikation im Internet kein Weg vorbei. Gegen diese Lösung werden von den Strafbehörden persönlichkeitsrechtliche Bedenken geltend gemacht. ${ }^{18}$ Zudem wird ar-

$15 \overline{\text { Donatsch Andreas, die öffentliche Verkündung }}$ des Strafurteils gemäss Konventionsrecht, in: Donatsch Andreas/Schmid Niklaus (Hrsg.), Strafrecht und Öffentlichkeit, Festschrift für Jörg Rehberg zum 65. Geburtstag, Zürich 1996, S. 132; Herzog Annelies, Öffentlichkeits- und Medienarbeit des Strafverteidigers (Litigation-PR), Diss. Univ. Zürich, Berlin 2014, S. 20; Riklin Franz, Öffentlichkeit des Strafverfahrens mit gegenläufiger Entwicklung: von der Marginalisierung der Hauptverhandlung zur Entheimlichung des Vorverfahrens, in: medialex 4/2008, S. 156.

16 BGE 124 IV 234 E. 3e; so auch Oberholzer (Fn. 6), Rz. 669.

17 ZR 1998 Nr. 42, 130; Bezgovsek Rok, Art. 6 Ziff. 1 EMRK und das steuerrechtliche Verfahren, Diss. Univ. Zürich 2002, S. 95; Donatsch (Fn. 15), S. 134; Riklin (Fn. 8), S. 118.

18 Wiprächtiger Hans, Kontrolle der Strafjustiz durch Medien und Öffentlichkeit - eine Illusion?, in: medialex 1/2004, S. 44 f.; Felber Markus, zur Anonymisierung von Gerichtsurteilen, in: SJZ 109/2013, S. 529, weist zu Recht darauf hin, dass bei der Online-Publikation von Entscheiden grössere Vorsicht geboten sei, als wenn Urteile akkreditierten Journalisten zugänglich gemacht werden. 
gumentiert, die vorgängige Anonymisierung der Entscheide verursache einen erheblichen Aufwand. ${ }^{19}$ Dieser unstreitig anfallende Mehraufwand ist allerdings nicht unverhältnismässig und im Interesse der Transparenz in Kauf zu nehmen.

Denkbar wäre die Publikation einer Liste aller anonymisierten Strafbefehle im Internet, ${ }^{20}$ während weiterhin vor Ort der vollständige Entscheid einsehbar ist. Um das Einsichtsrecht vor Ort zu ermöglichen, müsste die Internetpublikation zumindest die wichtigsten Eckpunkte des Strafbefehls enthalten. Diese Entscheidlisten müssten sodann in einer zentralen Datenbank publiziert werden, damit ersichtlich ist, wo der Strafbefehl eingesehen werden kann. Eine Publikation von Entscheidlisten im Internet ist auch für Einstellungs- und Nichtanhandnahmeverfügungen nötig, denn auch dort müssen Einsichtswillige überhaupt erst von solchen Verfahrenserledigungen Kenntnis erhalten. ${ }^{21}$

19 Vgl. dazu Hansjakob Thomas, Anmerkung zu Kantonsgericht St. Gallen, Anklagekammer, Entscheid vom 25. Mai 2011 i.S. S. gegen Staatsanwaltschaft St. Gallen - AK.2011.106-AK, in: forumpoenale 6/2011, S. 339; Riklin (Fn. 8), S. 121; siehe zur Anonymisierung von Entscheiden auch Schindler (Fn. 5), S. 753.

20 So auch der Vorschlag von Beutler Vera/Zehnder Regula, Gerichte tun sich schwer mit Transparenz, in: plädoyer 2/2011, S. 9; vgl. auch die Stellungnahme des Schweizer Presserates Nr. 25/2015.

21 Gless Sabine, Verfahrenserledigungen ohne Urteil: Pragmatismus und Gerechtigkeit, in: ZStrR 127 (2009), S. 391, schlägt demgegenüber vor, die Strafbehörden sollten regelmässig Einstellungsverfügungen von Verfahren veröffentlichen, an welchen ein berechtigtes öffentliches Interesse bestehe. Damit läge aber der Entscheid darüber, ob ein Entscheid dem Publikum zur Kenntnis zu bringen ist, bei den Strafbehörden selber, was unbefriedigend erscheint.
11 Mit dem Strafbefehlsverfahren als ressourcenschonendes und rasches Verfahren ist zwangsläufig eine gewisse Relativierung des Öffentlichkeitsgrundsatzes verbunden. ${ }^{22}$ Indes darf dieses Konzept nicht $\mathrm{zu}$ einer Aushebelung des Einsichtsrechts der Öffentlichkeit führen. Deshalb muss das nicht unbedenkliche Öffentlichkeitsdefizit dadurch ausgeglichen werden, dass nach Abschluss des Verfahrens Interessierte voraussetzungslos den Entscheid einsehen können. Um den Interessen der Betroffenen am Schutz ihrer Persönlichkeit genügend Rechnung zu tragen, wird die Einsichtnahme in aller Regel nur in den anonymisierten Entscheid gewährt. Die namentliche Identifizierung des Beschuldigten ist denn auch entbehrlich, um das Ziel der Schaffung von Transparenz über die Tätigkeit der Justiz zu erreichen. ${ }^{23}$

\section{Einstellungs- und Nichtanhandnahmeverfügungen}

12 Sowohl bei der Einstellung eines Strafverfahrens, als auch bei einer Nichtanhandnahme endet das Strafverfahren bereits in einem frühen Stadium. ${ }^{24}$ Infolge des Ausschlusses der Öffentlichkeit vom Vorverfahren finden diese Verfahren gänzlich unter Ausschluss der Öffentlichkeit statt. Zwar findet sich in der

22 Daphinoff Michael, das Strafbefehlsverfahren in der Schweizerischen Strafprozessordnung, Diss. Univ. Freiburg, Zürich 2012, S. 398 f.; Hutzler (Fn. 9), Rz. 143; Riklin in BSK StPO, vor Art. 352356, Rz. 1.

23 Vgl. zur Einsichtnahme in anonymisierte Entscheide sowie zum Zeitpunkt der Einsichtnahme interessierter Dritter in Strafbefehle i.E. Welte Eliane, Information der Öffentlichkeit über die Tätigkeit der Strafjustiz: Zur aktuellen Informationslandschaft und den Anforderungen an eine zeitgemässe Öffentlichkeitsarbeit der Strafbehörden, Diss. Univ. Zürich, Zürich/Basel/Genf 2016, S. $73 \mathrm{ff}$. und $66 \mathrm{ff}$.

24 Vgl. Art. 310 und Art. 319 StPO. 
Strafprozessordnung - bedauerlicherweise - keine Regelung zur Einsichtnahme in Einstellungs- und Nichtanhandnahmeverfügungen. ${ }^{25}$ Das Einsichtsrecht Dritter in solche Verfahrenserledigungen stützt sich indes auf ein Urteil des Bundesgerichtes, ${ }^{26}$ in welchem es festhielt, dass die Bevölkerung auch an Strafverfahren, welche ohne Verhängung einer strafrechtlichen Sanktion enden, ein legitimes Interesse haben kann. Die Verwehrung der Einsichtnahme in diese Entscheide wäre der Wahrnehmung des Strafverfahrens als transparentes Geschehen abträglich. ${ }^{27}$ Den entstehenden Mehraufwand aufgrund der Anonymisierung der Entscheide gilt es in Kauf zu nehmen, um dem Vorwurf einer intransparenten Geheimjustiz entschieden entgegenzutreten.

13 Dass die Bevölkerung ein Interesse an solchen Verfahren haben kann, hat sich am Fall des früheren Armeechefs Roland $\mathrm{Nef}^{28}$ gezeigt. Zu Recht bejahte das Bundesgericht ein gewichtiges öffentliches Interesse an der Frage, welches mutmassliche Verhalten des Amtsträgers zur Eröffnung eines Strafverfahrens geführt habe. Gemäss Bundesgericht ist die Be-

$25 \overline{\text { Solche Verfahrenserledigungen werden in Art. } 69}$ Abs. 2 StPO nicht genannt. Sodann findet auf Einstellungs- und Nichtanhandnahmeverfügungen auch Art. 84 StPO keine Anwendung, da dieser gemäss seinem klaren Wortlaut auf Sachurteile beschränkt ist. Ähnlich spricht auch Art. 6 Ziff. 1 EMRK nur von «Urteilen», welche öffentlich zu verkünden sind.

26 BGE 134 I 286.

27 BGE 137 I 16 E. 2.2; Bommer (Fn. 12), S 249; Oberholzer (Fn. 6), Rz. 668; Jäger Rolf, Strafuntersuchung und Medien im Spannungsfeld der Interessen unter besonderer Berücksichtigung der Zürcher Praxis, Diss. Univ. Zürich, Zürich/St. Gallen 2010, Rz. 47; Strebel Dominique, Geheimjustiz im Vormarsch, in: NZZ Nr. 64 vom 17.3.2011, S. 25.

28 BGE 137 I 16. grenzung der Einsichtnahme auf materielle Straferkenntnisse zu formalistisch. ${ }^{29}$ Indes verlangt es den Nachweis eines schutzwürdigen Informationsinteresses, welches gegen Geheimhaltungsinteressen abzuwägen sei. Dabei genüge es aber, wenn der Interessierte ein «ernsthaftes Interesse an der Kenntnisnahme» glaubhaft mache. ${ }^{30}$ Dieses ernsthafte Interesse ergibt sich ohne Weiteres aus der Kontrollfunktion der Öffentlichkeit gegenüber der Justiz. Von Bedeutung ist die Interessenabwägung allerdings beim Umfang der Einsichtsgewährung, denn voraussetzungslose Einsichtnahme bedeutet nicht zwingend auch umfangmässig unbeschränkte Einsichtnahme. Im Falle legitimer Geheimhaltungsinteressen der Betroffenen ist die Einsichtnahme nur in den anonymisierten und gegebenenfalls gekürzten Entscheid zu gewähren. ${ }^{31}$

14 Wird das Einsichtsrecht Dritter in solche Entscheide aus dem Prinzip der Justizöffentlichkeit abgeleitet, ist es widersprüchlich, für die Einsichtnahme zusätzliche Hürden zu errichten. ${ }^{22}$ Vielmehr muss die Einsicht voraussetzungslos ge-

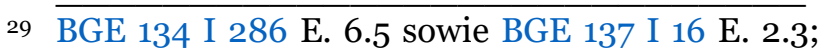
dieser Ansicht sind auch Gautschi Alain, Ambivalenz zwischen dem Grundsatz der Entscheidöffentlichkeit und dem Persönlichkeitsschutz der Beteiligten, in: Justice - Justiz - Giustizia 2013/1, Rz.19 sowie Jäger (Fn. 27), Rz. 41.

30 BGE 134 I 286 E. 5.1.

31 So auch Bommer (Fn. 12), S. 249: Die Schranken zulässiger Einsichtnahme müssen bei der schutzbedürftigen Person einsetzen, nicht beim Einsichtswilligen, weshalb in jedem Einzelfall eine Abwägung der aufeinandertreffenden Interessen vorgenommen werden muss, um den Umfang der Einsichtnahme zu bestimmen.

32 Bommer (Fn. 12), S. 248; Brunner Stephan, Einsichtnahme in Einstellungs- und Nichtanhandnahmeverfügungen, Anmerkungen zu BGE 134 I 286, in: medialex 3/2008, S. 145 . 
währt werden, 33 wobei in einem zweiten Schritt eine Interessenabwägung vorzunehmen ist, welche dazu führen kann, dass nur der anonymisierte oder gekürzte Entscheid zugänglich gemacht wird. 34 In Anbetracht der Bedeutung des Öffentlichkeitsprinzips spricht nichts dagegen, den Grundsatz der Entscheidöffentlichkeit im Strafverfahren auf alle verfahrensabschliessenden Entscheide auszudehnen. 35 Im Interesse der Rechtssicherheit sollte Art. 69 Abs. 2 StPO um die Kategorie der Einstellungs- und Nichtanhandnahmeverfügungen ergänzt werden.

\section{Die Medien als Vermittler von Informationen}

Medien sind omnipräsent und aus unserem Alltag nicht mehr wegzudenken. Indem die Medien - egal über welche Kanäle - Informationen zur Verfügung stellen, machen sie die Vorgänge im Gerichtssaal einem breiten Publikum zugänglich. Damit kommt ihnen die Funktion eines Bindeglieds zwischen Strafjustiz und Öffentlichkeit zu: ${ }^{36}$ So soll das wachsame

Vgl. die Kritik am geforderten Interessennachweis etwa bei Bommer (Fn. 12), S. 249; Brunner (Fn. 32), S. 145; Gautschi (Fn. 29), Rz. 24; Monnier Gilles, Anmerkung zu BGE 137 I 16, in: medialex 1/2011, S. 37.

34 BGE 137 I 16 E. 2.4.; Bommer (Fn. 12), S. 249; Gautschi (Fn. 29), Rz. 25; Schneider-Marfels Jascha, Einsichtnahme in behördliche Dokumente, Strafakten und Strafurteile, in: medialex 3/2014, S. 124.

35 Auch Häfelin Ulrich/Haller Walter/Keller Helen, Schweizerisches Bundesstaatsrecht, 8. Aufl., Zürich 2012, Rz. 856, sprechen sich für eine weitergehende Auslegung von Art. 30 Abs. 3 BV aus, um die Öffentlichkeit des Strafverfahrens besser zu gewährleisten.

36 BGE 113 Ia 309 E. 3c; BGE 129 III 529 E. 3.2; Herzog (Fn. 15), S. 46; Kuss (Fn. 3), S. 63; Schneider-Marfels (Fn. 34), S. 121; Stober Rolf, Medien als vierte Gewalt: zur Verantwortung der Massenmedien, in: Wittkämper Gerhard W. (Hrsg.), Medien und Politik, Darmstadt 1992, S. 29; Zeller Franz, Gerichtsöffentlichkeit als
Auge der Öffentlichkeit die Strafbehörden zur Sicherstellung eines korrekt ablaufenden Verfahrens anhalten. ${ }^{37}$ Wenngleich die Wirksamkeit dieser Kontrollfunktion umstritten ist, $3^{8}$ so kann doch zumindest die mediale Begleitung eines Strafprozesses Druck auf die Strafbehörden ausüben, sich um einen korrekten Verfahrensablauf zu bemühen. 39 Die Bildung von Vertrauen der Bevölkerung in die Strafjustiz bedingt, dass deren Tätigkeit für das Publikum transparent und nachvollziehbar gemacht wird.40 Nur ein kontinuierlicher Austausch zwischen Bevölkerung und Justiz vermag sicherzustellen, dass die Entscheide der Strafbehörden auf Akzeptanz stossen. Die Ausübung staatlicher Macht durch die Gerichte kann nur dann Legitimität bean-

Quelle der Medienberichterstattung, in: medialex 1/2003, S. 16.

37 BVerfGE 103, 44 (65), Guignard (Fn. 5), S. 55; Häfelin/Haller/Keller (Fn. 35), Rz. 856a; Müller/Schefer (Fn. 5), S. 964 f.; Saxer/Thurnheer in BSK StPO, Art. 69 Rz. 13; Schultz Hans, der Grundsatz der Öffentlichkeit im Strafprozess, in: SJZ 1973, S. 129; Zeller (Fn. 36), S. 15.

38 Siehe etwa Kuss (Fn. 3), S. 62; Martens Wolfgang, Öffentlichkeit als Rechtsbegriff, Habil. Univ. zu Münster, Bad Homburg 1969, S. 74 f.; Wettstein Edgar Jules, der Öffentlichkeitsgrundsatz im Strafprozess, Diss. Univ. Zürich, Basel/Zürich/Genf 1966, S. 65 f.; Zeller Franz, Medien und Hauptverhandlung - menschenrechtliche Leitplanken, in: Justice - Justiz - Giustizia 2006/1, Rz. 28.

39 Hauser Robert/Schweri Erhard/Hartmann Karl, Schweizerisches Strafprozessrecht, 6. Aufl., Basel 2005, § 52 Rz. 9; Heimgartner/Wiprächtiger in BSK BGG, Art. 59 Rz. 7; Schultz (Fn. 37), S. 131; Von Coelln Christian, zur Medienöffentlichkeit der Dritten Gewalt: rechtliche Aspekte des $\mathrm{Zu}-$ gangs der Medien zur Rechtsprechung im Verfassungsstaat des Grundgesetzes, Habil. Univ. Passau, Tübingen 2005, S. 196; Zeller Franz (Fn. 36), S. 16.

40 BGE 119 Ia 99 E. 4a; BGE 129 III 529 E. 3.2; Tschümperlin in BSK BGG, Art. 27 Rz. 3; Von Coelln (Fn. 39), S. 194; Wettstein (Fn. 38), S. 59; Zuck Rüdiger, Medien und Justiz, Notwendigkeit und Gefahren für die Rechtsprechung, in: DRiZ 1997, S. 26. 
spruchen, wenn die Strafjustiz deren Tätigkeit für das Publikum transparent und nachvollziehbar macht und sich der Kritik der Öffentlichkeit stellt. ${ }^{41}$

Damit kommt den Medien die essentielle Funktion zu, Öffentlichkeit herzustellen, indem sie Informationen vermitteln und zur Meinungsbildung der Bevölkerung beitragen..$^{2}$ Nicht zuletzt vermitteln sie dem Publikum Rechtskenntnisse, wobei damit nicht eine fundierte juristische Ausbildung der Bevölkerung gemeint sein kann. Vielmehr soll die Gerichtsberichterstattung die Bevölkerung verständlich mit grundlegenden Informationen darüber versorgen, wie Verstösse gegen die Rechtsordnung geahndet werden.43 Es kann dabei also lediglich, aber immerhin darum gehen, den Rechtsunterworfenen ohne juristische Fachausdrücke und komplexe Erläuterungen einen Einblick in die richterliche Entscheidfindung zu geben.44 Die Erreichung dieses Ziels wird indes durch die beschränkten Rechtskenntnisse eines Grossteils der Bevölkerung sowie den in

41 Baur Alex, auf dem Weg zur Kabinettsjustiz, in: plädoyer 2/2013, S. 12; Gless (Fn. 21), S. 390; Morsch Anke, Justiz und Medien, in: ZRP 2014, S. 254; Müller/Schefer (Fn. 5), S. 964; Wettstein (Fn. 38), S. 57 f.; Wiprächtiger (Fn. 18), S. 38; Zeller (Fn. 36), S. 16.

42 BVerfGE 97, 228 (267); Beater Axel, Medienrecht, Tübingen 2007, Rz. 21; Bergsdorf Wolfgang, die vierte Gewalt: Einführung in die politische Massenkommunikation, Mainz 1980, S. 76; Müller/Schefer (Fn. 5), S. 438; Paschke Marian, Medienrecht, 3. Aufl., Berlin 2009, Rz. 212; Saxer in BSK StPO, Art. 72 Rz. 1.

43 Fischli Ernst, die Öffentlichkeit der Justiz, erweiterter Text der Basler akademischen Antrittsrede vom 5.5.1970, in: BJM 1970, S. 58 f.; Franzki Harald, die Öffentlichkeit der Gerichtsverhandlung - was sie bezweckt, und wie sie missbraucht wird, in: DRiZ 1979, S. 82; Von Coelln (Fn. 39), S. 202 f.; Wettstein (Fn. 38), S. 93.

44 Bernhard (Fn. 3), S. 203; Rohner Max, Presse und Strafjustiz, in: ZStrR 88 (1972), S. 161; Wettstein (Fn. 38), S. 93 f., 129. der Medienberichterstattung herrschenden permanenten Zeitdruck erschwert.

\section{Strafjustiz und Medien - feindliche Brüder?}

17 Ohne die mediale Informationsvermittlung wäre es der Strafjustiz kaum möglich, das Vertrauen und die Akzeptanz der Rechtsunterworfenen zu gewinnen. 45 Zudem sind die Strafbehörden zuweilen auf die Medien angewiesen, etwa bei der Publikation von Fahndungsaufrufen. 46 Für die Medien wiederum stellt die Tätigkeit der Strafjustiz eine wichtige «Rohstoff-Quelle» der Berichterstattung dar, denn Strafprozesse stossen auf grosses Interesse. 47

18 Trotz der wichtigen Funktionen, welche die Medien bei der Information der Bevölkerung über Strafverfahren wahrnehmen, ist ihr Verhältnis zur Strafjustiz von Spannungen geprägt. Diese haben ihren Ursprung in den je eigenen Gesetz-

$45 \overline{\text { BGE } 129 \text { III } 529 \text { E. 3.2; Flehinghaus Otto, Justiz }}$ und Öffentlichkeit, in: DRiZ 1959, S. 165; Hassemer Winfried, grundsätzliche Aspekte des Verhältnisses von Medien und Strafjustiz, in: StV 2005, S. 167; Michlig Matthias, Öffentlichkeitskommunikation der Strafbehörden unter dem Aspekt der Amtsgeheimnisverletzung (Art. 320 StGB), Diss. Univ. Zürich, Zürich/Basel/Genf 2013, S. 23; Rohner (Fn. 44), S. 160 ff.; Saxer Urs, Öffentlichkeitsinformationen von Behörden im Rechtsstaat, in: medialex 1/2004, S. 26; Strebel (Fn. 4), S. $61 \mathrm{f}$.

46 Brunner Andreas, die verlorene Ehre der Medien!?, in: forumpoenale 2/2013, S. 93; Jäger (Fn. 27), Rz. 536 ff.; Michlig (Fn. 45), S. 19; Strebel (Fn. 4), S. 59 f.; Weiler Edgar, Medienwirkung auf das Strafverfahren, in: ZRP 1995, S. 134.

47 Friedrichsen Gisela, Strafverteidigung im Wandel - eine Aussenansicht, in: StV 2012, S. 632; KägiDiener Regula, Persönlichkeitsschutz im Verhältnis von Medien und Justiz, in: AJP 1994, S. 1103 f.; Schulz Uwe, die rechtlichen Auswirkungen von Medienberichterstattung auf Strafverfahren, Diss. Univ. Frankfurt a.M. 2002, S. 2; Wilmes Frank, über die Notwendigkeit von Public Relations in Strafprozessen, StraFo 2007, S. 11. 
lichkeiten und Interessenschwerpunkten der beiden Akteure Strafjustiz und Medien. ${ }^{8}$ Während die Strafjustiz häufig die teilweise stark reduzierte, auf das Skandalöse fokussierte Berichterstattung beklagt, wünschen sich die Medien von den Strafbehörden mehr Entgegenkommen und detailliertere Informationen. 49

Die Kommunikation von Strafjustiz und Medien über Strafverfahren unterscheidet sich wesentlich: Während die Strafjustiz rational und differenziert über ihre Tätigkeit Auskunft geben soll, steht es den Medien frei, sich der Emotionalisierung und Personalisierung zu bedienen sowie Inhalte $\mathrm{zu}$ überzeichnen und $\mathrm{zu}$ vereinfachen..$^{\circ}$ Eine sachliche und nüchterne Berichterstattung mag zwar der Wunschvorstellung der Strafbehörden entsprechen, lässt sich indes kaum mit

$48 \overline{\text { Aeschbach Daniel, Litigation-PR und der Court of }}$ Public Opinion, in: Justice - Justiz - Giustizia 2013/1， Rz. 31; Bernhard (Fn. 3), S. 207; Gostomzyk Tobias, die Rechtsrealität der Massenmedien, in: AfP 2005, S. 437 ff.; Hörisch Jochen, (wie) passen Justiz und Massenmedien zusammen?, in: StV 3/2005, S. 152 ff.; Jung Heike, (Straf-)Justiz und Medien, eine unendliche Geschichte, in: GA 5/2014, S. 265; Panier Christian/Jespers Jean-Jacques, justice, médias, pouvoir: un triangle infernal, Bruxelles 2004, S. 132; Schroers Jochen, versteckte Probleme bei der $\mathrm{Zu}-$ sammenarbeit zwischen Staatsanwaltschaften und Medien, in: NJW 1996, S. 969.

49 Dahs Hans, Handbuch des Strafverteidigers, 8. Aufl., Köln 2015, Rz. 100; Schmitt Bertram, der mediale Druck auf die Rechtsprechung hat zugenommen. ZRP-Rechtsgespräch (mit Rudolf Gerhardt), in: ZRP 2011, S. 222; Schroers (Fn. 48), S. 970.

50 Fischer Niklas S., die Medienöffentlichkeit im strafrechtlichen Ermittlungsverfahren, unter besonderer Berücksichtigung der Informationsfreiheitsgesetze, Diss. Univ. Berlin 2014, S. 35; Saxer (Fn. 45), S. 27; Schroers (Fn. 48), S. 970; Zabel Benno, «öffentlicher Pranger» und reformierter Strafprozess: aktuelle Tendenzen der Medialisierung vor und während des Ermittlungsverfahrens, in: GA 2011, S. 349. den Logiken und Zwängen vereinbaren, welchen die Medien unterliegen.

2o Gerade beim Strafprozess besteht eine erhöhte Gefahr von schädlichen Auswirkungen der medialen Begleitung, etwa einer massiven Prangerwirkung zu Lasten des Beschuldigten und einer medialen Vorverurteilung.51 Die Rahmenbedingungen der Medienberichterstattung führen zu einer Verschärfung dieser Gefahren: Der starke wirtschaftliche Wettbewerb in der Medienbranche zwingt die Medien zur Selektion und Kürzung von Inhalten, was Vereinfachungen und Verzerrungen in der Berichterstattung bewirken kann. ${ }^{2}$ Dabei folgen die Medien ihren eigenen Selektionsmechanismen, welche sich nicht mit den Interessen der Strafbehörden decken.53 Ein weiterer Kritikpunkt betrifft die teilweise mangelhafte Ausbildung der Journalisten: Aufgrund des Spardruckes in der Medienbranche verfügt in Grossteil der Print-

${ }_{51} \overline{\text { Bernhard (Fn. 3), S. 217; Beutler Vera, für den }}$ mutmasslichen Täter gilt die Unschuldsvermutung, die Medien und ihr Verhältnis zur Unschuldsvermutung in der Schweiz und England, Diss. Univ. Freiburg, Zürich/Basel/Genf 2013, Rz. 1052; Hauser/Schweri/Hartmann (Fn. 39), $\S 52$ Rz. 15; Müller Gerda, Probleme der Gerichtsberichterstattung, in: NJW 2007, S. 1617; Oberholzer (Fn. 6), Rz. 643; Redeker Konrad, Anwalt und Presse, in: AnwBl 1961, S. 301; Schneider Hans Joachim, das Geschäft mit dem Verbrechen: Massenmedien und Kriminalität, München 1980, S. 130 f.; Stellungnahme des Schweizer Presserates Nr. 25/2015, E. 1.

52 BVerfGE 103, 44 (67); Bernhard (Fn. 3), S. 207; Beutler (Fn. 51), Rz. 1395; Flehinghaus (Fn. 45), S. 165; Michlig (Fn. 45), S. 30; Studer Peter, was dürfen Richter und Journalisten voneinander erwarten?, in: AJP 2005, S. 1447; Zuck Rüdiger, Medien und Justiz, Notwendigkeit und Gefahren für die Rechtsprechung, in: DRiZ 1997, S. 23.

53 Beater (Fn. 42), Rz. 930; Branahl (Fn. 4), S. 77 ff.; Danziger Christine, die Medialisierung des Strafprozesses, eine Untersuchung zum Verhältnis von Medien und Strafprozess, Diss. Univ. Berlin 2009, S. 241; Riklin Franz, Schweizerisches Presserecht, Bern 1996, § 6 Rz. 74. 
medien über keinen spezialisierten Gerichtsberichterstatter mehr. 54

\section{Medienpräsenz der Verfahrensbeteiligten}

21 Zuweilen versuchen die Verfahrensbeteiligten selber, die mediale Plattform für ihre Zwecke zu nutzen. Denn einen Prozess gilt es nicht nur im Gerichtssaal zu gewinnen, sondern auch in der Wahrnehmung des Publikums, dem «Gerichtshof der Öffentlichkeit».55 Bemühungen, die Meinung der Öffentlichkeit mittels offensiver Medienarbeit zu beeinflussen, gewinnen in Anbetracht der zunehmend intensiven medialen Begleitung von Strafverfahren an Bedeutung. ${ }^{56}$ Der Grund für den Gang an die Medien ist naheliegend: Wer diesen Schritt als Erster wagt, sieht gute Chancen, die Meinungshoheit für sich in Anspruch $\mathrm{zu}$

54 Gullotti Franco/Binz Roland, im Gerichtssaal der Öffentlichkeit, in: Anwaltsrevue 9/2010, S. 359; Herzog (Fn. 15), S. 75; Schmitt (Fn. 49), S. 221; Sprecher Margrit, die Gerichtsberichterstattung, in: Heer Marianne/Urwyler Adrian (Hrsg.), Justiz und Öffentlichkeit, Bern 2007, S. 84 f.; Zuck (Fn. 52), S. 24; Zülch Christoph, Justiz und Öffentlichkeitsarbeit, in: DRiZ 1994, S. 37.

55 Aeschbach (Fn. 48), Rz. 4; Binz Roland, folgenschwere Urteile im Gerichtsaal der Öffentlichkeit, in: der Bund vom 7.5.2010, S. 12; Friedrichsen Gisela/Gerhardt Rudolf, Werbung für die Gerechtigkeit - oder für was?, in: DRiZ 2012, S. 75; Gostomzyk Tobias, Anwälte wissen, wie Prozesse gewonnen werden - und wie gewinnt der Mandant die öffentliche Meinung?, in: AnwBl 2008, S. 587; Gullotti/Binz (Fn. 54), S. 363; Wolff Uwe, Medienarbeit für Rechtsanwälte, ein Handbuch für die effektive Kanzlei-PR, Wiesbaden 2010, S. 172.

56 Berger Nikolaus, Fordert prozessbegleitende Krisen-PR die Strafjustiz neu heraus?, in: DRiZ 2012, S. 70; Clemen Peter, Litigation-PR - die neue Wunderwaffe, um Prozesse zu gewinnen?, in: DRiZ 2010, S. 117; Gatzweiler Norbert, Medienberichterstattung und hieraus resultierende Verteidigungsmöglichkeiten, in: StraFo 1995, S. 64 . nehmen.57 Gerade bei aufsehenerregenden Strafverfahren mit prominenter Beteiligung kommt es vor, dass die beschuldigte Person einen prominenten PR-Berater engagiert mit dem Ziel, ihren Gesichtsverlust durch aktive Kommunikation über die Medien möglichst gering $\mathrm{zu}$ halten. Indes sind solche Medienstrategien nicht unproblematisch, weil Medienauftritte kontraproduktiv wirken und einen Glaubwürdigkeitsverlust bewirken können. $5^{8}$

22 Gelegentlich treten auch Mitglieder der Strafbehörden von sich aus an die Medien, um diese für ihre Zwecke zu nutzen. 59 Solchen Medienauftritten sind indes relativ enge Grenzen gesetzt, indem die Strafbehörden durch die Strafprozessordnung zu einer die Unschuldsvermutung und die Reputation des Beschuldigten wahrenden Kommunikation verpflichtet sind.6o In Anbetracht der Rolle der Strafjustiz als objektive und unabhängige Behörde sind eigennützige Me-

$57 \overline{\text { Freuding Stefan, die Verlagerung von Strafver- }}$ fahren in Massenmedien - ein pessimistischer Ausblick, in: ZRP 2010, S. 161.

58 Becker Florian, die aktive Öffentlichkeitsarbeit von Staatsanwaltschaften und das Grundrecht auf informationelle Selbstbestimmung, in: Livonius Barbara et al. (Hrsg.), Strafverteidigung im Wirtschaftsleben - Festgabe für Hanns W. Feigen zum 65. Geburtstag am 13. März 2014, Köln 2014, S. 16 f.; Herzog (Fn. 15), S. 145 f.; Hürlimann Brigitte, «Die verlorene Ehre der Medien!?», Interesse der Strafverfolgung und der Parteien «contra» Anspruch der Medien auf Information, in: forumpoenale 2/2013, S. 101.

59 Friedrichsen (Fn. 47), S. 632; Jung (Fn. 48), S. 262; Oberholzer (Fn. 6), Rz. 656; Tilmann Job, Prozessführung der Staatsanwaltschaft und Medien, in: StV 2005, S. 175 .

60 Becker (Fn. 58), S. 17 f.; Berger (Fn. 56), S. 70; Meyer Myrna, der Gerichtsprozess in der medialen Berichterstattung: die Macht der mediengeprägten öffentlichen Meinung und die Rolle der Prozessbeteiligten in der heutigen Mediengesellschaft, Diss. Univ. Hamburg, Baden-Baden 2014, S. $260,279 \mathrm{ff}$. 
dienauftritte mit Vorsicht zu geniessen. Würden sich Vertreter der Strafjustiz in der öffentlichen Diskussion erkennbar auf eine Seite schlagen und damit in einem konkreten Fall plötzlich nach aussen hin als Partei in Erscheinung treten, wäre dies der Objektivität und Unabhängigkeit der Strafjustiz abträglich. ${ }^{61}$ Folglich ist die Grenze des Zulässigen dort zu ziehen, wo die eigenen Darstellungsinteressen der Strafbehörden im Vordergrund stehen und sie ein Verfahren in ihrem Sinn $\mathrm{zu}$ beeinflussen versuchen.

Wachsender Beliebtheit erfreut sich das Phänomen der sog. «Litigation-PR», also der gezielte Einsatz von Kommunikationsmitteln durch die Verteidigung mit der Absicht einer gezielten Einwirkung auf die öffentliche Meinung. ${ }^{62}$ Ziel ist es, den Mandanten in ein besseres Licht zu rücken und ihn vor einem Reputationsverlust zu bewahren. Denn wie ein Beschuldigter von der Öffentlichkeit wahrgenommen wird, hängt nicht allein davon ab, ob der Prozess mit einer Verurteilung oder einem Freispruch endet. Vielmehr bringt bereits die Involvierung

61 Meyer (Fn. 60), S. 289; Tschümperlin in BSK BGG, Art. 27 Rz. 29; Wiprächtiger Hans, Öffentlichkeit und Justiz, in: Ehrenzeller Bernhard/Saxer Urs (Hrsg.), St. Galler Tagung zur Öffentlichkeitskommunikation des Staates, St. Gallen 2010, S. 153; Winsauer Günther, Öffentlichkeitsarbeit der Justiz, in: österreichische Juristenkommission (Hrsg.), Recht und Öffentlichkeit: 40 Jahre österreichische Juristenkommission, Wien 2004, S. 143.

62 Aeschbach (Fn. 48), Rz. 13; Berger (Fn. 56), S. 72; Danziger (Fn. 53), S. 240; Freuding (Fn. 57), S. 160; Herzog (Fn. 15), S. 22; Holzinger Stephan/Wolff Uwe, im Namen der Öffentlichkeit: Litigation-PR als strategisches Instrument bei juristischen Auseinandersetzungen, Wiesbaden 2009, S. 8; Meyer (Fn. 60), S. 251; Schmidheiny Andrea, Wahrnehmung der Litigation-PR aus der Perspektive der Zürcher Zivil- und Strafrechtspflege, in: Justice - Justiz - Giustizia 2013/4, Rz. 2. in ein Strafverfahren einen Reputationsverlust mit sich. ${ }^{63}$ Folglich tun die Betroffenen gut daran, möglichst früh ihre eigene Sichtweise darzulegen und die Öffentlichkeit für sich zu gewinnen. Indes gibt es für solche Bemühungen keine Erfolgsgarantie, denn auch die beste Litigation-PR vermag ein einmal beschädigtes Image eines Beschuldigten in der öffentlichen Wahrnehmung - selbst im Falle eines Freispruchs - nicht mehr vollständig zu korrigieren. ${ }^{64}$ Eine erfolgreiche aktive Medienstrategie der Verteidigung bedingt Kenntnisse im Umgang mit den Medien, denn im schlechteren Fall kann Litigation-PR die gegenteilige Wirkung des Erhofften erzielen. In der Medienwelt herrschen andere Regeln als vor Gericht und wer die medialen Gegebenheiten $\mathrm{zu}$ wenig kennt, hat viel zu verlieren, aber nur wenig zu gewinnen. ${ }^{65}$

\section{Mediale Einflüsse auf das Strafverfahren}

24 Die Existenz von Einflüssen der Berichterstattung auf das Strafverfahren ist im Grundsatz unbestritten. Indes gehen die Meinungen mit Bezug auf die Zwänge im Einzelnen und die damit verbundenen

63 Dahs Hans, der Anwalt im Strafprozess, in: AnwBl 1959, S. 180; Ernst Stefan, Kameras im Gerichtssaal, in: ZUM 1996, S. 190; Schlüter Oliver, Verdachtsberichterstattung: zwischen Unschuldsvermutung und Informationsinteresse, Diss. Univ. Berlin, München 2011, S. 2 f., 25 f.; Schneider (Fn. 51), S. 131; Wilmes (Fn. 47), S. 12.

64 Berger (Fn. 56), S. 74; Quoirin Marianne, Litigation-PR, in: DRiZ 2012, S. 87; Wegner Carsten, Sanktionsrechtliche Krisen-PR: Gedanken eines Strafverteidigers, in: Hellmann Uwe/Schröder Christian (Hrsg.), Festschrift für Hans Achenbach, Heidelberg 2011, S. $582 \mathrm{f}$.

65 Gostomzyk (Fn. 55), S. 587; Dahs (Fn. 49), Rz. 100; Gatzweiler (Fn. 56), S. 64; Herzog (Fn. 15), S. 150 ff.; Maier Nadine, Litigation PR: Medienarbeit in juristischen Auseinandersetzungen, Hamburg 2012, S. 11; Wegner (Fn. 54), S. 593 . 
Gefahren auseinander.66 Unbestritten dürfte sein, dass auch mögliche Kehrseiten des Öffentlichkeitsgrundsatzes im Strafverfahren dessen grundlegende Bedeutung in einem demokratischen Rechtsstaat nicht in seinen Grundfesten zu erschüttern vermögen. ${ }^{67}$

Zum einen besteht die Gefahr eines zunehmenden Zwanges zu «mediengerechtem» Auftreten der Verfahrensbeteiligten. Die Annahme liegt nahe, dass die Beteiligten im Bewusstsein um die breitflächige mediale Verbreitung der Geschehnisse im Gerichtssaal ihr Handeln und Aussageverhalten anpassen. ${ }^{68}$ Des Weiteren droht eine Beeinträchtigung der Wahrheitsfindung, indem sich die Beteiligten wegen möglicher Reputationsschäden durch die Berichterstattung davon abhalten lassen könnten, sich vor Gericht frei zu äussern. ${ }^{69}$ Insbesondere durch Rundfunkaufnahmen aus dem Gerichtssaal könnten sodann Zeugen vorab

$66 \overline{\text { Hinsichtlich der Wirkungen der medialen Be- }}$ richterstattung auf das Strafverfahren und deren Folgen fehlen bis anhin umfassende und aussagekräftige empirische Untersuchungen.

67 Wie Fischli (Fn. 43), S. 55 f., zutreffend festhält, muss daher das Ziel darin bestehen, die Nachteile des Konzepts der Öffentlichkeit im Strafverfahren «mit einem klug dosierten System von Remedien unter Kontrolle $\mathrm{zu}$ bringen oder wenigstens $\mathrm{zu}$ mildern.»

68 BVerfGE 103, 44 (69); Barrelet Denis, Justiz oder Zirkus?, in: medialex 4/2002, S. 173; Burbulla Frank, die Fernsehöffentlichkeit als Bestandteil des Öffentlichkeitsgrundsatzes, Diss. Univ. Bochum, Frankfurt a.M. 1998, S. 129; Danziger (Fn. 53), S. 341; Eckertz-Höfer Marion, Fernsehöffentlichkeit im Gerichtssaal, in: DVBl 7/2012, S. 390 f.; Franzki (Fn. 43), S. 82; Friedrichsen/Gerhardt (Fn. 55), S. 76; Sarstedt Werner, Rundfunkaufnahmen im Gerichtssaal, in: JR 1956, S. 123.

69 BGHSt 9, 280 (283); Britz Guido, Fernsehaufnahmen im Gerichtssaal, ein rechtsvergleichender Beitrag zum Öffentlichkeitsgrundsatz im Strafverfahren, Diss. Univ. Saarbrücken, BadenBaden 1999, S. 265 ff.; Michlig (Fn. 45), S. 76. über die Geschehnisse im Gerichtssaal informiert werden. ${ }^{70}$ Gegenwärtig ist das Anfertigen von Bild- und Tonaufnahmen im Gerichtssaal verboten (vgl. Art. 71 Abs. 1 StPO). In Anbetracht des Gefahrenpotenzials von audiovisuellen Aufnahmen muss dieses Verbot auch weiterhin bestehen bleiben. ${ }^{71}$ Die Aufrechterhaltung dieses Verbots drängt sich aufgrund der spezifischen Charakteristika dieser Informationskanäle auf: Zum einen erlauben audiovisuelle Aufnahmen eine Information Dritter praktisch ohne zeitliche Verzögerung, also in Echtzeit. Zum anderen fehlen durch diese sofortige Informationsvermittlung jegliche $\mathrm{Si}$ cherheitsnetze; so können einmal verbreitete Falschinformationen im Nachhinein kaum mehr richtiggestellt werden. ${ }^{2}$

26 Zu glauben, die Strafjustiz gehe ihrer Tätigkeit frei von äusseren Einflüssen nach, wäre geradezu naiv. Auch die Strafbehörden werden in ihrer Tätigkeit von vielen äusseren Faktoren beeinflusst.73

70 Flehinghaus (Fn. 45), S. 165; Franzki (Fn. 43), S. 82; Vogel Irmela, Fernsehübertragungen von Strafverfahren in der Bundesrepublik Deutschland und in den USA, Diss. Univ. Bielefeld, Frankfurt a.M. 2005, S. 86; Von Coelln (Fn. 39), S. 433; Witzler Jochen, die personale Öffentlichkeit im Strafverfahren, Diss. Univ. Heidelberg, Pfaffenweiler 1993, S. 162.

71 Vgl. dazu i.E. Welte (Fn. 23), S. $231 \mathrm{ff}$.

72 Pampalk Madalena/Raab Stephanie/Scheickl Nicole, Richter und der Umgang mit Medien, in: österreichische Richterzeitung (RZ) 2014, S. 31; Schellkopf Holger, Alles zu seiner Zeit. Von der Zeitung zur Echtzeit, in: Schröder Michael/Schwanebeck Axel (Hrsg.), live dabei - Echtzeitjournalismus im Zeitalter des Internets, Baden-Baden 2014, S. $102 \mathrm{f}$.

73 Gatzweiler (Fn. 56), S. 64; Kepplinger Hans M./Zerback Thomas, der Einfluss der Medien auf Richter und Staatsanwälte, in: Hestermann Thomas (Hrsg.), von Lichtgestalten und Dunkelmännern - wie die Medien über Gewalt berichten, Wiesbaden 2012, S. 161; Ryter Marianne, Justiz und Öffentlichkeit - ein paar Gedanken, 
Problematisch wird es dann, wenn sich die urteilenden Richter bereits im Vorfeld der Urteilsfällung einer unsachlichen und mit Angriffen auf ihre Person verbundenen medialen Hetzjagd ausgesetzt sehen.74 Wichtig ist, dass sich die Strafbehörden dieser Risiken bewusst sind und sich mit ihnen auseinandersetzen, anstatt sie zu negieren.75 Daneben sehen sich die Richter auch mit anderen gesellschaftlichen Einflüssen wie etwa politischen Werturteilen konfrontiert. ${ }^{76}$ Von den Strafbehörden zu verlangen, sich jeglichen potenziellen äusseren Einflüssen gänzlich zu entziehen, wäre weder möglich noch erstrebenswert. Den Richtern muss zugetraut werden, sich gegenüber äusseren Einflüssen genügend abzugrenzen. 77

in: Justice - Justiz - Giustizia 2008/4, Rz. 5; Wagner Joachim, Strafprozessführung über Medien, Baden-Baden 1987, S. 90; Zimmerli Ulrich, wenn die Politik Druck macht - Richtertätigkeit unter Beeinflussungsversuchen, in: Justice - Justiz - Giustizia 2009/4, Rz. 9.

74 BGE 113 Ia 309 E. 5a; Dahs (Fn. 53), S. 179; Müller Georg/Thommen Marc, Unabhängigkeit versus Öffentlichkeit der Justiz, in: Heer Marianne/Urwyler Adrian (Hrsg.), Justiz und Öffentlichkeit, Bern 2007, S. 36; Walter Hans Peter, Gedanken zum Richteramt, in: ZBJV 1991, S. $630 \mathrm{f}$.

75 Aeschbach (Fn. 48), Rz. 54; Boehme-Nessler Volker, unabhängige Richter in der Mediengesellschaft? Überlegungen zum Einfluss der Medien auf die Gerichte, in: AfP 2010, S. 542; Clemen (Fn. 55), S. 117; Danziger (Fn. 53), S. 427; Pfeiffer Gerd, die innere Unabhängigkeit des Richters, in: Fürst Walther/Herzog Roman/Umbach Dieter C. (Hrsg.), Festschrift für Wolfgang Zeidler, Berlin 1987, S. 73 .

76 BGE 105 Ia 157 E. 6a; Noll Alfred J., Justiz und mediale Öffentlichkeit, in: Noll Alfred J. (Hrsg.), Justiz unter Druck? Zur Rolle der dritten Gewalt in Österreich, Wien/New York 1991, S. 69 f.; Ryter (Fn. 73), Rz. 5; Wiprächtiger Hans, der Strafrichter und die Massenmedien, in: plädoyer 3/2000, S. 29.

77 BGE 105 Ia 157 E. 6a; Aeschbach (Fn. 48), Rz. 48; Boehme-Nessler (Fn. 75), S. 542; Caesar Peter, richterliche Unabhängigkeit und öffentliche Meinung, in: DRiZ 1994, S. 457; Jäger (Fn. 27),
27 Schliesslich kann die mediale Berichterstattung über Strafverfahren eine Verletzung der Unschuldsvermutung bewirken. Nicht selten kommt es - nebst der ebenfalls stigmatisierend wirkenden öffentlich zugänglichen Hauptverhandlung zu einer verstärkten Blossstellung des Beschuldigten. ${ }^{78}$ Insbesondere eine Berichterstattung über dessen persönliche Verhältnisse kann seine Privatsphäre und die Unschuldsvermutung empfindlich verletzen. ${ }^{79}$ Die Medien verschreiben sich zuweilen vorschnell dem Ziel, den Beschuldigten belastende Umstände ans Licht zu bringen, und sind kaum bereit, ihren Standpunkt zu revidieren, sollte sich der Tatverdacht nicht erhärten. ${ }^{80}$

28 Darüber hinaus kann die mediale Berichterstattung über Strafverfahren für die Betroffenen mit einer starken Prangerwirkung und Erschwerung der Resozialisierung verbunden sein. ${ }^{81}$ Wird der Beschuldigte in den Medien als Schuldiger präsentiert und über Wochen inten-

Rz. 174; Pfeiffer (Fn. 75), S. 73; Riklin (Fn. 1), S. 69.

78 Beutler (Fn. 51), Rz. 1052; Dahs (Fn. 53), S. 180; Hünig Markus, Probleme des Schutzes des Beschuldigten vor den Massenmedien, Diss. Univ. Zürich 1973, S. 45; Redeker (Fn. 51), S. 301; Schneider (Fn. 51), S. 130 f.

79 BGE 129 III 532 E. 3.2; Dalbkermeyer Birgit, der Schutz des Beschuldigten vor identifizierenden und tendenziösen Pressemitteilungen der Ermittlungsbehörden, Diss. Univ. Bonn, Frankfurt a.M. 1994, S. 11 f.; Oberholzer (Fn. 6), Rz. 674.

80 Bornkamm Joachim, die Berichterstattung über schwebende Strafverfahren und das Persönlichkeitsrecht des Beschuldigten, in: NStZ 1983, S. 103; Hünig (Fn. 78), S. 45; Schmid Ulrich, Freispruch für Wulff, in: NZZ Nr. 49 vom 28.2.2014, S. 4.

81 Fröhling Mareike, der moderne Pranger: von den Ehrenstrafen des Mittelalters bis zur Prangerwirkung der medialen Berichterstattung im heutigen Strafverfahren, Diss. Univ. Kiel, Marburg 2014, S. 315 ff.; Riklin (Fn. 53), § 6 Rz. 60; Schultz (Fn. 37), S. 132; Stellungnahme des Schweizer Presserates Nr. 25/2015, E. 1. 
siv über den Fall berichtet, wird seine Involvierung in ein Strafverfahren in den Köpfen des Publikums verankert. ${ }^{82}$ Sodann kann durch die Berichterstattung auch das Umfeld des Beschuldigten von dessen Involvierung in ein Strafverfahren Kenntnis erhalten, was dessen Reintegration in die Gesellschaft erschwert. ${ }^{83}$

Die dargestellten Zwänge sind - so problematisch sie auch sein mögen - in zweierlei Hinsicht zu relativieren: Zum einen betreffen sie primär eine überschaubare Anzahl besonders aufsehenerregender Strafverfahren. Ebenso wenig dürfen diese potenziellen Zwänge darüber hinwegtäuschen, dass die mediale Berichterstattung nur einen von vielen äusseren Faktoren darstellt, welche auf das Strafverfahren wirken. ${ }^{84}$ Wird der Freiheit der medialen Berichterstattung ein hoher Stellenwert beigemessen, lassen sich diese Zwänge nicht gänzlich ausschliessen. ${ }^{85}$ Indes sollten sich die Strafbehörden dieser potenziellen Beeinflussungen bewusst sein. Mit geeigneten Massnahmen können Druckversuche von aussen reduziert werden, etwa mittels eines Verbotes von Bild- und Tonaufnahmen aus dem Gerichtssaal (vgl. Art. 71 StPO).

82 Bernhard (Fn. 3), S. 217; Fröhling (Fn. 81), S. 328 ff.; Keil Nadine K., Verdachtsberichterstattung: Medienberichterstattung über Straftatverdächtige, Diss. Univ. Marburg, Frankfurt a.M. 2013, S. 67; Schneider (Fn. 51), S. 131.

83 Bornkamm (Fn. 80), S. 103.

84 BGE 105 Ia 157 E. 6a; Riklin (Fn. 1), S. 69; Wiprächtiger (Fn. 76), S. 28 f.; Zeller Franz, zwischen Vorverurteilung und Justizkritik: verfassungsrechtliche Aspekte von Medienberichten über hängige Gerichtsverfahren, Diss. Univ. Bern 1998, S. 127.

85 Dieser Ansicht ist auch Widmaier Gunter, Gerechtigkeit - Aufgabe von Justiz und Medien?, in: NJW 2004, S. 403.

\section{Informationsbeschaffung der Bevölkerung über Strafverfahren}

3o Bei der Beschaffung von Informationen über Strafverfahren durch die Bevölkerung dürften die Presse, Radio und Fernsehen zu den wichtigsten Medien zählen. ${ }^{86}$ In den letzten Jahren gewann indes das World Wide Web als Informationsquelle unaufhaltsam an Bedeutung. 87 Meldungen über Strafverfahren auf den Webseiten der Tageszeitungen sind weit verbreitet. Häufig sind solche OnlineArtikel mit einer Kommentarfunktion versehen, welche sich grosser Beliebtheit erfreut.88 Zuweilen wird Interessierten gar die Möglichkeit geboten, einen Strafprozess im Internet mit minimaler zeitlicher Verzögerung mitzuverfolgen - anhand eines sog. Live-Tickers, bei welchem im Minutentakt mittels kurzer Meldungen über das Geschehen im Gerichtssaal informiert wird. ${ }^{89}$ Bei der Entwicklung der Berichterstattung über Strafverfahren fallen insbesondere drei Tendenzen ins Auge:

86

87 und Medienformate, in: Bonfadelli Heinz/Jarren Otfried/Siegert Gabriele (Hrsg.), Einführung in die Publizistikwissenschaft, 3. Aufl., Bern 2010, S. 460 ff.: Die Beliebtheit dieses Mediums ist unter anderem auf dessen tiefe Zugangsschranken sowie den gegenüber der klassischen Presse höheren Aktualitätsgrad zurückzuführen.

88 Fröhling (Fn. 81), S. 162; Roesler Alexander, bequeme Einmischung. Internet und Öffentlichkeit, in: Münker Stefan/Roesler Alexander (Hrsg.), Mythos Internet, 2. Aufl., Frankfurt a. M. 2010, S. 181; Springer Nina, suche Meinung, biete Dialog? Warum Leser die Kommentarfunktion auf Nachrichtenportalen nutzen, in: Wolling Jens/Will Andreas/Schumann Christina (Hrsg.), Medieninnovationen, Konstanz 2012, S. 248.

89 Schellkopf (Fn. 72), S. 102; ein aktuelles Beispiel für einen ausführlichen Live-Ticker zu einem Strafprozess ist jener im Fall des bekannten Komikers Karl Dall (Urteil DG 140208 des Bezirksgerichts Zürich vom 9.12.2014). 
31 Zum einen gewinnt der Wunsch der Medienkonsumenten, ihre eigene Meinung zu den vermittelten Informationen kundzutun und sich in die Diskussion einzubringen, an Bedeutung. Dies zeigt sich an der Nutzung der Kommentarfunktion auf den Online-Portalen der Tageszeitungen. 90

Des Weiteren ist eine stetige Zunahme der Geschwindigkeit der Informationsverbreitung festzustellen - insbesondere durch das Internet.91 Die negativen Folgen dieser immer schnelleren Verbreitung von Informationen sind unübersehbar: Selbst die kleinste Publikation im Internet kann sich schnell zu einem medialen Fegefeuer entwickeln ${ }^{92}$ und einmal publizierte falsche Informationen können im Nachhinein kaum mehr richtiggestellt werden.93 Das Diktat der Ak-

$9 0 \longdiv { \text { Fisch Martin/Gscheidle Christoph, Mitmachnetz } }$ Web 2.0: rege Beteiligung nur in Communitys. Ergebnisse der ARD/ZDF-Onlinestudie 2008, in: Media Perspektiven 7/2008, S. 356; Maletzke Gerhard, Ziele und Wirkungen der Massenkommunikation, Grundlagen und Probleme einer zielorientierten Mediennutzung, Hamburg 1976, S. 134 f.; Mergel Ines et al., Praxishandbuch: soziale Medien in der öffentlichen Verwaltung, Wiesbaden 2013, S. $24 \mathrm{f}$.

91 Beutler (Fn. 51), Rz. 250; Gullotti/Binz (Fn. 54), S. 360; Hürlimann Brigitte, die Gerichte manövrieren sich zusehends in die Isolation, in: Blum Roger (Hrsg.), eingeschüchterte Richter? Instrumentalisierte Medien? Journalismus und Justiz im Dialog, Solothurn 2017, S. 67; Puttenat Daniela, Praxishandbuch Presse- und Öffentlichkeitsarbeit, eine Einführung in professionelle PR und Unternehmenskommunikation, Wiesbaden 2007, S. 16.

92 Gullotti/Binz (Fn. 54), S. 360; Müntinga Maren, die journalistischen Wahrheits- und Sorgfaltspflichten und die Möglichkeiten ihrer Durchsetzung, eine Untersuchung anhand der Landesmediengesetze, Diss. Univ. Giessen, Baden-Baden 1999, S. 24.

93 Dahs (Fn. 49), Rz. 98; Russ-Mohl Stephan, ohnmächtige Helden der vierten Gewalt, in: NZZ Nr. 148 vom 30.6.2015, S. 50; Schellkopf (Fn. 72), S. 102; vgl. dazu als aktuelles Beispiel: Wie unse- tualität der Informationsvermittlung verträgt sich schlecht mit den Zielen des Strafprozesses, welcher der Aufklärung und Sanktionierung von Normverstössen in einem förmlichen Verfahren dient.94

33 Als dritte Entwicklung ist eine zunehmende Emotionalisierung und Personalisierung der Berichterstattung über Strafverfahren zu beobachten. Indem Handlungen einer bestimmten Person zugeschrieben werden und diese dadurch ein Gesicht erhält, wird versucht, die Aufmerksamkeit der Mediennutzer zu gewinnen. $95 \mathrm{Zu}$ diesem Zweck wird in den Medien häufig ein Bild des Beschuldigten präsentiert, um den Adressaten ein Bild zur Story zu liefern. ${ }^{6}$ Eine andere Entwicklung betrifft die Gerichtsentscheide, die zunehmend weniger als Entscheide des Gerichts als Ganzes wahrgenommen werden. Stattdessen gerät zunehmend der einzelne Richter in den Fokus des Interesses. Als Konsequenz daraus nimmt der Druck der Öffentlichkeit auf die Richter zu.97 Zudem eignen sich Strafverfahren sehr gut, um mittels Betonung des Spektakulären die Inhalte auf Dauer im Gedächtnis des Publikums zu verankern. Durch die genannten medialen Darstel-

re falsche Eilmeldung zum NPD-Urteil zustande kam.

94 BVerfGE 103, 44 (66); Danziger (Fn. 54), S. 356 f.; Hörisch (Fn. 48), S. 153; Panier/Jespers (Fn. 48), S. $132 \mathrm{f}$.

95 BVerfGE 101, 361 (390); Beater (Fn. 43), Rz. 926; Beutler (Fn. 51), Rz. 1396 ff.; Danziger (Fn. 53), S. 220; Gostomzyk (Fn. 48), S. 438; Strebel Marcel, Spannungsfeld «Polizei und Medien», in: Kriminalistik 2008, S. 61.

96 Beater (Fn. 42), Rz. 923; Boehme-Nessler Volker, BilderRecht, die Macht der Bilder und die Ohnmacht des Rechts: wie die Dominanz der Bilder im Alltag das Recht verändert, Berlin / Heidelberg 2010, S. 138 ff.; Gullotti/Binz (Fn. 54), S. 360.

97 Boehme-Nessler (Fn. 96), S. 147; Gostomzyk (Fn. 48), S. 438 f.; Tschümperlin in BSK BGG, Art. 27 Rz. 31. 
lungsstrategien kann die Konzentration auf das Wesentliche verloren gehen, indem an die Stelle einer sachlichen und fundierten Information des Publikums eine auf das Spektakuläre fokussierte Sensationsberichterstattung tritt, durch welche der Informationsgehalt leicht Schaden nimmt.

\section{Ausblick}

34 Die Medienlandschaft wandelt sich kontinuierlich, womit sich mit Bezug auf die Berichterstattung über Strafverfahren neue Fragen stellen. Bereits seit längerer Zeit diskutiert wird die Anfertigung audiovisueller Aufnahmen im Gerichtssaal, wie sie in der Schweiz gegenwärtig verboten (Art. 71 Abs. 1 StPO), in den Vereinigten Staaten von Amerika hingegen Gang und Gäbe ist bei aufsehenerregenden Strafprozessen. Die Konsequenzen einer Zulassung solcher Aufnahmen lassen sich nur grob erahnen, weshalb grösste Vorsicht geboten ist, sollen nicht die Grundpfeiler eines fair und möglichst frei von äusseren Druckversuchen ablaufenden Gerichtsverfahrens gefährdet werden. 98

35 Ähnliche Gefahren bergen auch neuere mediale Erscheinungen wie das Twittern und Online-Live-Ticker aus dem Gerichtssaal: Aufgrund der Informationsvermittlung praktisch in Echtzeit entfallen jegliche Sicherheitsnetze und eine einmal kommunizierte Falschinformati-

98 Kritisch etwa Barrelet (Fn. 68), S. 173; Dahs (Fn. 63), S. 181; Deutscher Richterbund (DRB), Stellungnahme zu einer Verfassungsbeschwerde der n-tv Nachrichtenfernsehen GmbH \& Co. KG, in: DRiZ 1996, S. 247 f.; Eckertz-Höfer (Fn. 68), S. 390 f.; Franzki (Fn. 43), S. 82; Fröhling (Fn. 81), S. 287 ff.; Schmitt (Fn. 49), S. 222; Schultz (Fn. 37), S. 134 sowie Wettstein (Fn. 38), S. $107 \mathrm{ff}$. on kann kaum mehr richtiggestellt werden.99 Bezüglich Twittermeldungen und Online-Live-Ticker aus dem Gerichtssaal besteht gegenwärtig keine gesetzliche Regelung. Eine solche Berichterstattung ist grundsätzlich zulässig, kann aber aus sitzungspolizeilichen Gründen (vgl. Art. 63 StPO) untersagt werden. ${ }^{100} \mathrm{Da}$ sich eine Abschätzung der Gefahren im Einzelfall aber als schwierig erweist, sollte die Frage der Zulässigkeit einer Online-Live-Berichterstattung aus dem Gerichtssaal einer einheitlichen rechtlichen Regelung zugeführt werden. ${ }^{101}$ Durch die spezifischen Gefahren der möglichen Vorabinformation später aussagender Zeugen sowie der fehlenden Sicherheitsnetze aufgrund der Verbreitung der Informationen in Echtzeit mittels einer solchen textbasierten Berichterstattung werden zentrale Grundpfeiler eines fairen und korrekt ablaufenden Gerichtsverfahrens gefährdet. Eine Zulassung textbasierter Berichterstattung aus dem Gerichtssaal im Einzelfall erscheint wenig praktikabel, zumal sich die konkrete Gefährdung nur schwer im Voraus quantifizieren lässt. In Anbetracht der auf dem Spiel stehenden Interessen an einem korrekt ablaufenden Verfahren und einer Wahrheitsfindung frei von unsachgemässen äusseren Einflüssen erscheint die Statuierung eines entsprechenden Verbotes sowohl erforderlich als auch verhältnismässig. Diskutiert wird sodann

99 Pampalk/Raab/Scheickl (Fn. 72), S. 31; Schellkopf (Fn. 72), S. $102 \mathrm{f}$.

100 Art. 71 Abs. 1 StPO spricht von «Bild- und Tonaufnahmen» und ist daher nicht einschlägig; Saxer/Thurnheer in BSK StPO, Art. 71 Rz. 7; Von Coelln Christian, Justiz und Medien, in: AfP 3/2014, S. 202.

${ }^{101}$ So auch Fanti Sébastien, De l'utilisation de Twitter lors des audiences publiques des tribunaux, in: medialex 1/2011, S. 2, der eine klare Regelung der Zulässigkeit des Twitterns anregt. 
auch die Direktübertragung der öffentlichen Beratungen des Bundesgerichts via Internet. Allerdings ist diese Idee auf Widerstand gestossen, und der Nutzen solcher Übertragungen erscheint zweifelhaft. ${ }^{102}$

\section{VI. Öffentlichkeitsarbeit der Strafjustiz}

36 In Anbetracht der meist leeren Zuschauerränge im Gerichtssaal und der daraus resultierenden Brückenfunktion der Medien als Informationsvermittler ${ }^{103}$ gewinnt auch die Informationstätigkeit der Strafbehörden an Bedeutung. Denn nur wenn die Strafjustiz die dafür unabdingbaren Informationen zu Verfügung stellt, können die Medien faktenbasiert und fundiert über Strafverfahren berichten. ${ }^{104}$

102 Motion «Live-Stream-Direktübertragung öffentlicher Urteilsberatungen des Bundesgerichts» (13.3660), eingereicht von Martin Schmid am 21.6.2013; Felber Markus, mit Gruppendynamik zur Einstimmigkeit, in: plädoyer 1/2014, S. 68; Freiburghaus Dieter, «Live-stream» am Bundesgericht - mehr Transparenz oder erster Schritt zum «Court-TV»?, in: Justice - Justiz - Giustizia 2013/4, Rz. 2 ff.; Hodel Peter, Direktübertragung der Urteilsberatungen am Bundesgericht und richterliche Unabhängigkeit, in: Justice - Justiz - Giustizia 2013/4, Rz. 1 f.; Schubarth Martin, öffentliche Urteilsberatung, in: Donatsch Andreas/Schmid Niklaus (Hrsg.), Strafrecht und Öffentlichkeit, Festschrift für Jörg Rehberg zum 65. Geburtstag, Zürich 1996, S. 309; von Graffenried Alec in AB-NR 2014, S. 233; kritisch auch Schindler (Fn. 5), S. $750 \mathrm{f}$.

103 BGE 113 Ia 309 E. 3c; Bührke Horst, Plädoyer für die Öffentlichkeitsarbeit der Justiz, in: DRiZ 1966, S. 10; Dahs (Fn. 49), Rz. 97; Herzog (Fn. 15), S. 46; Jung (Fn. 48), S. 260 f.; Kuss (Fn. 3), S. 63; Wettstein (Fn. 38), S. 65 f.; Zuck (Fn. 40), S. 28.

104 Brunner (Fn. 46), S. 95; Huff Martin, Information der Öffentlichkeit ist auch eine Aufgabe der Gerichte, überarbeitete und ergänzte Fassung eines Vortrages gehalten vor der Juristischen Gesellschaft zu Berlin am 17. Januar 1996, Berlin / New York 1996, S. 207; Meyer (Fn. 60), S. 263; Pampalk/Raab/Scheickl (Fn. 72), S. 31; Schmidheiny Andrea, die Umsetzung des Öffentlich-
Will die Strafjustiz Einfluss auf das Bild nehmen, welches in der Öffentlichkeit von ihr gezeichnet wird, muss sie ihre während langer Zeit von Zurückhaltung geprägte - Kommunikationsstrategie überdenken. Denn: Berichtet wird über jeden, also auch über denjenigen, welcher die Medien meidet. ${ }^{105}$ Im Laufe der letzten Jahre ist eine aktive Kommunikationstätigkeit mehr und mehr zu einer Bringschuld staatlicher Akteure geworden. In Anbetracht der immer zahlreicheren medialen Informationskanäle sowie der zunehmenden Beschleunigung der Informationsverbreitung genügt es nicht mehr, wenn die Strafjustiz bloss reaktiv zur Berichterstattung über ihre Tätigkeit Stellung nimmt. ${ }^{106}$

37 Bei der Öffentlichkeitsarbeit der Strafjustiz geht es darum, mittels transparenter Kommunikation zum richtigen Zeitpunkt Vertrauen und Verständnis für ihre Tätigkeit zu wecken, Akzeptanz zu erzeugen sowie Spannungen und Widerstände aus dem Weg zu schaffen. ${ }^{107}$ Kurz: Die Justiz

keitsprinzips am Zürcher Obergericht und an den Bezirksgerichten, in: Justice - Justiz - Giustizia 2012/2, Rz. 17; Strebel (Fn. 4), S. 61; Zülch (Fn. 5), S. 36.

105 Danziger (Fn. 53), S. 426; Riklin (Fn. 1), S. 69; Ryter (Fn. 73), Rz. 24; Strebel (Fn. 4), S. 62 f.; Thiesmeyer Heinrich, Öffentlichkeitsarbeit der Justiz, in: DRiZ 1964, S. 73; Von Coelln (Fn. 39), S. 516; wie Schindler (Fn. 5) zutreffend feststellt, steht die aktive Kommunikation aufgrund der Stellung der Justiz zwar nicht im Vordergrund; nichtsdestotrotz sind Fälle denkbar, in welchen eine aktive Informationstätigkeit geboten ist.

106 Hassemer Winfried, Medien im Bundesverfassungsgericht, in: Rode Irmgard/Leipert Matthias (Hrsg.), das moderne Strafrecht in der Mediengesellschaft: Einfluss der Medien auf das Strafverfahren, München 1990, S. 17; Hürlimann (Fn. 58), S. 102; Ryter (Fn. 73), Rz. 16; Thiesmeyer (Fn. 105), S. 74; Tschümperlin in BSK BGG, Art. 27 Rz. 3.

107 Danziger (Fn. 53), S. 426; Rota Franco P., Public Relations und Medienarbeit: effektive Öffentlichkeitsarbeit von Unternehmen im Informations- 
muss sich verständlich machen und verhindern, dass in der Öffentlichkeit ein negatives oder unzutreffendes Bild von ihrem Wirken entsteht. Um Transparenz gegenüber der Öffentlichkeit zu schaffen, ist es unabdingbar, auch Fehler und Schwächen offen $\mathrm{zu}$ thematisieren. ${ }^{108}$ Nicht zuletzt geht es darum, die korrekte Wahrnehmung der Strafbehörden sowie ihrer Stellung und Aufgaben $\mathrm{zu}$ fördern. ${ }^{109}$ Ziel und Zweck von Öffentlichkeitsarbeit der Strafjustiz ist also nicht, dass der Richter nach der Urteilsberatung den Entscheid erläutern und rechtfertigen muss. ${ }^{110}$ Vielmehr geht es darum, dass - idealerweise durch einen Medienbeauftragten - sachlich, objektiv und wertungsfrei über die Entscheide informiert und Unklarheiten beseitigt wer-

zeitalter, 2. Aufl., München 2002, S. 77 ff.; Saxer Urs, Justizkommunikation im Rechtsstaat, in: Heer Marianne/Urwyler Adrian (Hrsg.), Justiz und Öffentlichkeit, Bern 2007, S. 51; Wassermann Rudolf, Probleme aktiver Öffentlichkeitsarbeit der Justiz, in: Wassermann Rudolf (Hrsg.), Justiz und Medien, Neuwied 1980, S. 149; Wettler Peter M., die 3. Gewalt und die Medien, in: Heer Marianne/Urwyler Adrian (Hrsg.), Justiz und Öffentlichkeit, Bern 2007, S. 119; Zülch (Fn. 54), S. 36.

108 Joerger Gernot, Öffentlichkeitsarbeit, Stuttgart 1975, S. 12; Litzka Gerhard, Verhältnis Justiz und Medien, in: Noll Alfred J. (Hrsg.), Justiz unter Druck? zur Rolle der dritten Gewalt in Österreich, Wien/New York 1991, S. 74; Strebel (Fn. 95), S. 63; Wassermann (Fn. 107), S. $151 \mathrm{f}$.

109 Bührke Horst, Plädoyer für die Öffentlichkeitsarbeit der Justiz, in: DRiZ 1966, S. 7; Feik Rudolf, öffentliche Verwaltungskommunikation: Öffentlichkeitsarbeit, Aufklärung, Empfehlung, Warnung, Habil. Univ. Salzburg, Wien 2007, S. 312 f.; Litzka (Fn. 108), S. 74; Pleil Gerhard J., Öffentlichkeitsarbeit: ein Weg zum Unternehmenserfolg - Möglichkeiten und Grenzen vom PublicRelations, Stuttgart 1977, S. 11 f.; Wassermann (Fn. 107), S.155.

110 Stellungnahme des DRB (Fn. 98), Pampalk/ Raab/Scheickl (Fn. 72), S. 31; Spühler Karl, Gericht und Medien - Erfahrungen, in: ZBJV 1994, S. 554; Von Coelln (Fn. 48), S. 520; diff. Gerhardt Rudolf, Medienauskunft in eigener Sache?, in: ZRP 2008, S. 135. den. ${ }^{111}$ Wichtig ist dabei insbesondere die Bemühung, die gefällten Urteile möglichst auch für die juristisch nicht geschulte Bevölkerung verständlich zu kommunizieren. ${ }^{112}$ Es ist unumgänglich, dass die Justizorgane ihre frühere Abwehrhaltung gegenüber den Medien ablegen, sich mit den Eigengesetzlichkeiten der Medien auseinandersetzen und sich um eine transparente Informationspolitik bemühen. ${ }^{113}$ Dabei geht es nicht zuletzt darum, Öffentlichkeitsarbeit nicht bloss als leidiges Übel zu betrachten, sondern die Vorteile einer aktiven Kommunikation mit der Öffentlichkeit zu nutzen. Denn je früher die Strafjustiz von sich aus über ihre Tätigkeit informiert, desto eher kann sie die Berichterstattung in ihrem Sinn beeinflussen. ${ }^{114}$

38 Eine zeitgemässe Öffentlichkeitsarbeit stellt eine kontinuierlich zu betreibende Aufgabe dar, denn die Beziehungen zur Öffentlichkeit bedürfen stetiger Pflege,

111 Stellungnahme des DRB (Fn. 98); Spühler (Fn. 110), S. 554; Tschümperlin in BSK BGG, Art. 27 Rz. 30.

112 Felber Markus, Justiz als Nonvaleur?, in: NZZ Nr. 148 vom 29.6.2013, S. 13; Prinz Matthias, Justiz und Medienöffentlichkeit, in: Prinz Matthias/ Peters Butz (Hrsg.), Medienrecht im Wandel, Festschrift für Manfred Engelschall, BadenBaden 1996, S. 251; Ryter (Fn. 73), Rz. 12, 19; Thiesmeyer (Fn. 105), S. 73.

113 BVerwGE 104, 105 (108 ff.); Danziger (Fn. 53), S. 427; Hürlimann (Fn. 58), S. 102; Riklin (Fn. 1), S. 69; Ryter (Fn. 73), Rz. 24; Saxer (Fn. 105), S. 50 f.; Wassermann Rudolf, Notwendigkeit und Grenzen der Justizkritik, in: DRiZ 1966, S. 13.

114 Brunner (Fn. 46), S. 95; Kottkamp Julia, Öffentlichkeitsarbeit von Staatsanwaltschaften in der Mediengesellschaft, eine repräsentative Studie, Wiesbaden 2015, S. 41; Meyer (Fn.60), S. 263; Rüttimann Jean-Paul, Jalons pour une communication active des tribunaux!, in : AJP 2005, S. 1453 ; Saxer (Fn. 107), S. 51; Strebel (Fn. 4), S. 62 f.; Zülch (Fn. 54), S. 36. 
damit Vertrauen entstehen kann. ${ }^{115}$ Besonderes Augenmerk ist bei der Informationstätigkeit auf den Zeitfaktor zu legen: Nur wenn die Strafjustiz zeitnah informiert, kann sie das Vertrauen der Rechtsunterworfenen gewinnen und die Berichterstattung in ihrem Sinne beeinflussen. Anderenfalls kann sie nur noch zuschauen, wie andere ein - möglicherweise negatives - Bild von ihrer Tätigkeit zeichnen. ${ }^{116}$ Die veränderten Anforderungen an die Öffentlichkeitsarbeit der Justiz erfordern eine verstärkte Professionalisierung in Form einer fachlich kompetenten Person, welche die nötigen Informationen innert nützlicher Frist kommunizieren kann.117 Die Informationsmittel sind vielfältig - zu denken ist etwa an Medienmitteilungen, Medienkonferenzen, Interviews, Aussprachen mit Medienvertretern sowie die Präsenz im Internet.118 Im Vordergrund stehen dürfte indes die Einrichtung einer Medienstelle, wie sie je länger je mehr an vielen grösseren Gerichten besteht. ${ }^{119}$

115 Hünig (Fn. 78), S. 50; Riklin (Fn. 1), S. 71; Rüttimann (Fn. 114), S. 1452; Spühler (Fn. 110), S. 55; Strebel Dominique, das Ende der Omerta in der Justiz, in: Justice - Justiz - Giustizia 2013/1, Rz. 21; Wassermann (Fn. 107), S. 157; Wettstein (Fn. 38), S. 121.

116 Gullotti/Binz (Fn. 54), S. 360; Jäger (Fn. 27), Rz. 691; Maier (Fn. 65), S. 58 f.; Rota (Fn. 107), S. $39 \mathrm{ff}$.

117 Bührke (Fn. 103), S. 6; Danziger (Fn. 53), S. 426 f.; Jäger (Fn. 27), Rz. 691; Joerger (Fn. 108), S. 37 f.; Pleil (Fn. 109), S. 43 f.; Prinz (Fn. 112), S. 251; Spühler (Fn. 110), S. 554; Thiesmeyer (Fn. 105), S. 74; Wassermann (Fn. 107), S. 174 f.; Zülch (Fn. 54), S. 36 f.

118 Joerger (Fn. 108), S. 63 ff.; Kottkamp (Fn. 114), S. 142 ff.; Prinz (Fn. 112), S. 252; Rohner (Fn. 44), S. 149 ff.; Von Coelln (Fn. 39), S. 515; Wasserman (Fn. 107), S. $161 \mathrm{ff}$.

119 Stellungnahme des DRB (Fn. 98); Pampalk/Raab/ Scheickl (Fn. 72), S. 31; Rüttimann (Fn. 114), S. 1453; Strebel (Fn. 115), Rz. 7.

\section{Der Fall «Carlos» oder: Wie man es nicht machen sollte}

39 Während es mit einer aktiven, adäquaten Öffentlichkeitsarbeit der Strafbehörden viel $\mathrm{zu}$ gewinnen gibt, kann eine nur halbherzig betriebene oder fehlende Kommunikation die Verbreitung von Spekulationen fördern und dem Vertrauen in die Strafjustiz schaden. ${ }^{120}$ Zudem besteht die Gefahr, dass sich die Journalisten ihre Informationen auf Umwegen beschaffen. ${ }^{121}$ Aus der Sicht der Strafjustiz ist es aber von grosser Wichtigkeit, dass die medial verbreiteten Informationen über Strafverfahren der Wahrheit entsprechen und die Bevölkerung objektiv informiert wird. Die negativen Folgen einer vernachlässigten Informationspolitik der Strafjustiz treten mit der stetigen Beschleunigung der Kommunikationsflüsse in aller Deutlichkeit zutage: Was einmal den Weg in die Berichterstattung gefunden hat, verbreitet sich unter Umständen wie ein Lauffeuer und löst mithin eine mediale Dynamik aus, welche nicht mehr kontrollierbar ist. Auf diese Weise kann eine nicht faktenbasierte und stark subjektiv geprägte öffentliche Diskussion entstehen, welcher die Strafjustiz nur hilflos zusehen kann. ${ }^{122}$ Die Verhin-

120 Flehinghaus (Fn. 45), S. 165; Jung (Fn. 48), S. 263; Strebel (Fn. 115), Rz. 4; Wiprächtiger Hans, Bundesgericht und Öffentlichkeit: zum Verhältnis von Medien und Justiz, in: Mieth Dietmar/Pahud de Mortanges René (Hrsg.), Recht - Ethik - Religion, der Spannungsbogen für aktuelle Fragen, Festgabe für Bundesrichter Guisep Nay zum 60. Geburtstag, Luzern 2002, S. 11.

121 Friedrichsen (Fn. 47), S. 632; Jäger (Fn. 27), Rz. 712; Keil (Fn. 82), S. 257; Spühler (Fn. 110), S. 556; Strebel (Fn. 4), S. 61; Wettstein (Fn. 38), S. 121.

122 Bührke (Fn. 103), S. 6; Jäger (Fn. 27), Rz. 691; Joerger (Fn. 108), S. 15; Maier (Fn. 65), S. 58 f.; Rota (Fn. 107), S. 39 ff. 
derung solcher medialer «Shitstorms » ${ }^{123}$ erfordert von der Strafjustiz, aktiv statt nur reaktiv zu agieren.

40 Wie wichtig eine transparente - und zeitnahe! - Informationspolitik der Strafbehörden ist, hat sich jüngst im schweizweit Aufsehen erregenden Fall «Carlos» in aller Deutlichkeit gezeigt. ${ }^{24}$ Während die Medien den Fall bereits breitflächig ausschlachteten, hüllten sich die Vertreter der Strafjustiz in eisernes Schweigen. Jegliche Stellungnahme von offizieller Seite blieb zunächst aus und die Medien brachten nach und nach weitere Details der Geschichte ans Licht. Erst zwölf Tage nach Bekanntwerden des Falles gab es eine Pressekonferenz - eindeutig zu spät, wie sich später herausstellte. ${ }^{125}$ Doch damit nicht genug: Die zuständigen Behördenvertreter schoben sich vor aller Augen gegenseitig die Schuld an den begangenen Fehlern zu. Dieses ungeschickte Agieren der Verantwortlichen schadete

$123 \overline{\text { Fechner Frank, Medienrecht: Lehrbuch des ge- }}$ samten Medienrechts unter besonderer Berücksichtigung von Presse, Rundfunk und Multimedia, 16. Aufl., Tübingen 2015, 12. Kapitel Rz. 243: Der Begriff «Shitstorm» bezeichnet kollektive Missfallenskundgebungen, bspw. in den Social Media.

$124 \mathrm{Zu}$ den Hintergründen des Falles siehe Urteil 6B_85/2014 des BGer vom 18.2.2014; Jositsch Daniel/Aebersold Peter/Schweizer Caroline, der Fall Carlos: Chronik und Analyse einer Tragödie, in: Jusletter vom 16. Dezember 2013; Ninck Matthias, der Verrat - wie funktionieren Journalisten und Politiker? Und was sagt ihr Zusammenspiel über unsere Gesellschaft aus? Der Fall Carlos ist ein Lehrstück über die Angst vor den Medien, in: das Magazin Nr. 10 vom 8.3.2014, S. $8 \mathrm{ff}$.

125 Vgl. dazu Gasser Benno, Schluss mit der Luxusbehandlung, in: NZZ Nr. 207 vom 7.9.2013, S. 16; Minor Liliane, Justizdirektor ohne Juristengeschwurbel, in: Tagesanzeiger Nr. 62 vom 16.3.2015, S. 17 . der Glaubwürdigkeit der Justiz massiv. ${ }^{126}$ In Anbetracht der fehlenden Kommunikationsstrategie der Verantwortlichen erstaunt es nicht, dass die Öffentlichkeit mit Unverständnis und Empörung reagierte und dass eine polemische, von Halbwahrheiten geprägte öffentliche Diskussion in Gang kam, welche in einem veritablen medialen «Shitstorm» gipfelte. ${ }^{127}$ Die heftige Reaktion der Öffentlichkeit ist die logische Folge der fehlenden Transparenz seitens der Strafjustiz und deren Versuch, den Informationsanspruch der Öffentlichkeit zu negieren. ${ }^{128}$ Wäre nicht viel zu lange mit der ersten Pressekonferenz zugewartet worden und hätte einer der zuständigen Behördenvertreter die Verantwortung übernommen und klar Position bezogen, wäre es kaum zu einem wochenlangen medialen Shitstorm dieses Ausmasses gekommen.

\section{Ein Öffentlichkeitskonzept tut not}

41 Gerade die wenigen, besonders aufsehenerregenden Strafprozesse wären die beste Gelegenheit, um den Medien und der Öffentlichkeit zu zeigen, dass die Strafjustiz bereit ist, in einen offenen Dialog zu treten und Transparenz zu schaffen.129 Im Spannungsfeld zwischen Per-

126 Baur Alex, der Dorfkönig, in: Die Weltwoche Nr . 11/2014 vom 12.3.2014, S. 32 f.; Minor (Fn. 125), S. 17; Ninck (Fn. 124), S. 8 ff.

127 So auch Minor Liliane, «Zickzackkurs bis zum Schluss», in: Tagesanzeiger Nr. 140 vom 20.6.2014, S. 15 und Schürer Andreas, «Mir geht es gegen den Strich», in: NZZ Nr. 50 vom 1.3.2014, S. 18.

128 So auch Minor (Fn. 127), S. 15.

129 Diese Gelegenheit wurde nicht nur im Fall «Carlos», sondern auch im Prozess gegen den bekannten deutschen Wettermoderator Jörg Kachelmann nicht genutzt. Vielmehr wurde in diesem Strafprozess die mediale Hetzjagd durch den exzessiven Ausschluss der Öffentlichkeit von den Befragungen noch zusätzlich angeheizt; vgl. dazu 
sönlichkeitsschutz der Verfahrensbeteiligten und Informationsanspruch der Öffentlichkeit muss die Strafjustiz abwägen, inwieweit dem Informationsbedürfnis der Öffentlichkeit Rechnung getragen werden kann. Ein adäquates Konzept für den Umgang mit dem öffentlichen Informationsinteresse verlangt zwar eine sorgfältige Planung, ist aber im Ergebnis die bessere Lösung als ein unprofessionelles und planloses Auftreten nach aussen, welches die Strafbehörden immer wieder einem Rechtfertigungszwang aussetzt. Zu meinen, die Strafjustiz könne die Kontrolle darüber erlangen, was und wie über ihre Tätigkeit berichtet wird, wäre vermessen. Nichtsdestotrotz ist es wichtig und richtig, dass sie von sich aus informiert und $\mathrm{zu}$ einem guten Dialog mit der Öffentlichkeit beiträgt. Eine aktive Öffentlichkeitsarbeit der Justiz stellt die notwendige Ergänzung einer fundierten Gerichtsberichterstattung dar, welche die Tätigkeit der Justiz kritisch begleitet. 130

Boehme-Nessler Volker, Kachelmann-Prozess: am Ende nur Verlierer, in: Legal Tribune Online vom 31.5. 2011.

130 Saxer (Fn. 107), S. 77: «Mit einer aktiven Öffentlichkeitsarbeit der Justiz können die zentralen Werte des Rechtsstaates kommuniziert werden: Unabhängigkeit, Unparteilichkeit Sachlichkeit, Rechtsbezogenheit, Transparenz und Fairness. Justizkommunikation ist in besonderem Mass Kommunikation des Rechtsstaates im Rechtsstaat. (...) Geschwätzig muss die Justiz deswegen nicht werden.» 